\title{
Coherent Long Reach OIDMA-PONs Enabled By Electronic Dispersion Compensation
}

\author{
Eslam A. El-Fiky ${ }^{1}$, Ziad A. El-Sahn ${ }^{1}$, and Hossam M. H. Shalaby ${ }^{2}$ \\ ${ }^{1}$ Electrical Engineering Department,Faculty of Engineering,Alexandria University, Alexandria 21544, Egypt \\ Email:eslam_a_fiky@yahoo.com,ziad.elsahn@ieee.org \\ ${ }^{2}$ Department of Electronics and Communications Engineering Egypt-Japan University of Science and Technology \\ Alexandria 21934,Egypt Email: shalaby@ieee.org
}

\begin{abstract}
We present a coherent optical interleave-division multiple-access (OIDMA) technique with electronic dispersion compensation that can be employed in next generation long reach PONs with large number of users at a reasonably low launch power.
\end{abstract}

(C) 2013 Optical Society of America

OCIS codes: $060.0060,060.4510$.

\section{Introduction}

Passive optical networks (PONs) have been deployed all over the world as the last mile broadband access technology. Current gigabit PONs and 10G-PONs employ time-division multiplexing (TDM) to share the bandwidth among different users [1]. Increasing bandwidth requirements with the new emerging services pushes TDM-PONs to its capacity due to higher complexity. Next generation PONs (NG-PONs) are envisioned to support larger number of users, bandwidth, and distance coverage. Recently, different multiple access techniques have been proposed for NG-PONs, e.g., wavelength-division multiplexing (WDM) [1], optical code-division multiple-access (OCDMA) [2], orthogonal frequecny-divison multiple-access (OFDMA) [3], and optical interleave-division multiple-access (OIDMA) [4, 5].

In this paper, we introduce a novel coherent detection OIDMA-PON uplink scenario with electronic dispersion compensation as shown in Fig. 1. OIDMA uses user specific interleavers as the only means for user separation [6]. Information bits from the optical network unit (ONU) $k \in\{1,2, \ldots, K\}$, denoted by $\left\{b^{(k)}\right\}$, are spread using low rate spreading codes. This generates a coded sequence $\left\{c_{j}^{(k)}\right\}$, then a chip level user specific interleaver is applied to produce the transmitted sequence $\left\{x_{j}^{(k)}\right\}$ which then modulates the optical carrier using quadrature phase shift keying (QPSK) modulation. Uplink signals coming from different users are combined at the remote node and passed to the feeder fiber till the optical line terminal (OLT). The optical signal is then coherently detected, dispersion is electronically compensated, and the output is applied to the OIDMA receiver. The OIDMA receiver is an iterative receiver that utilizes the soft decoding (turbo) algorithm [6].

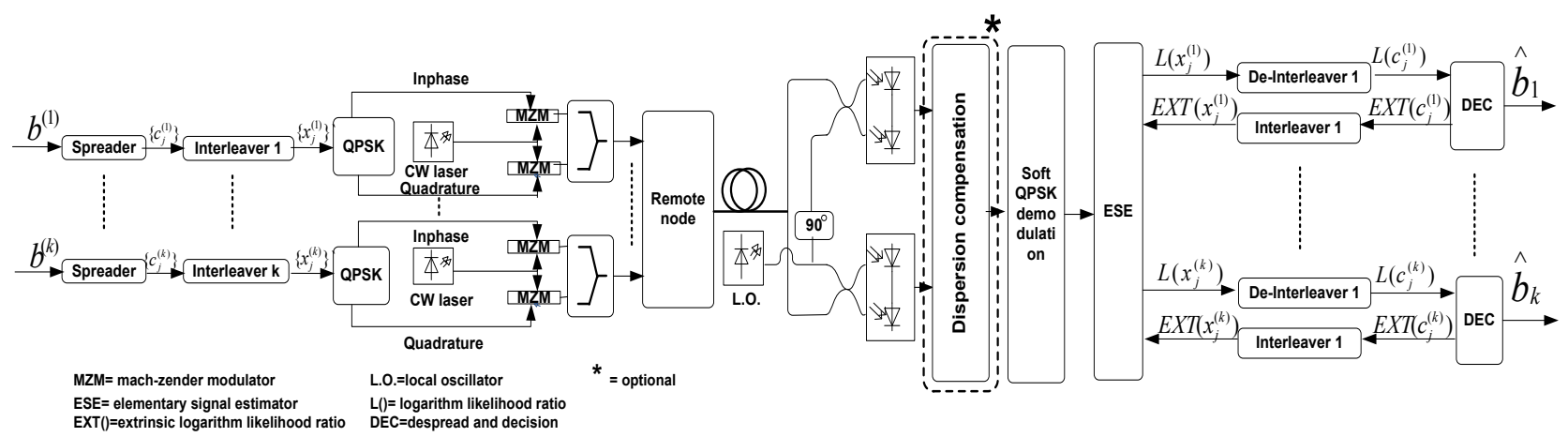

Fig. 1. Architecture of the proposed coherent OIDMA-PON with electronic dispersion compensation (uplink direction). 


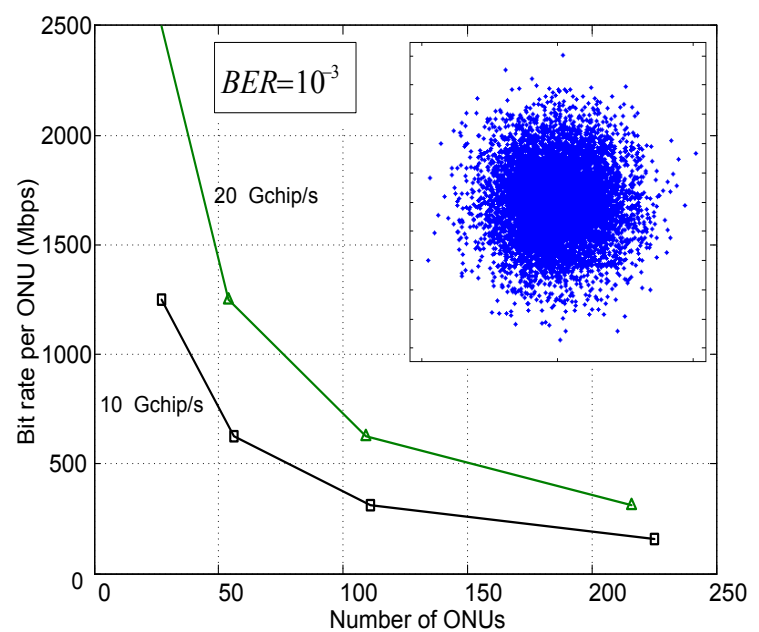

(a) User bit rate versus number of ONUs at 10 and $20 \mathrm{Gchip} / \mathrm{s}$ for 100 $\mathrm{km}$ reach with electronic dispersion compensation. The inset shows the constellation diagram of the received signal for 100 users at -5 $\mathrm{dBm}$ launch power.

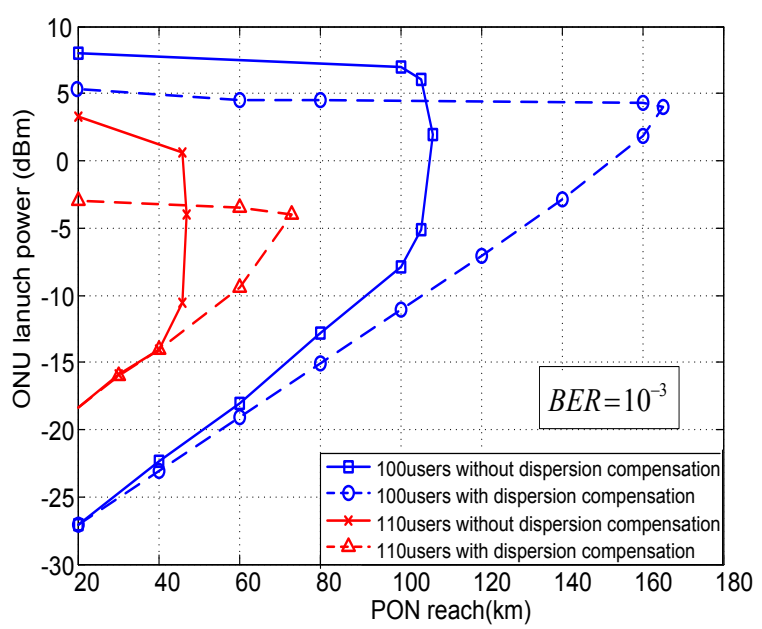

(b) ONU launch power versus PON reach for 100 and 110 users at $10 \mathrm{Gchip} / \mathrm{s}$ with and without dispersion compensation.

Fig. 2. Uplink performance of the proposed coherent OIDMA-PON.

\section{Performance of the proposed coherent OIDMA-PON system}

In this section, we report the performance of the proposed system in Fig. 1, through a Matlab/Optisystem cosimulation. Fig. 2a shows user bit rate versus the number of ONUs for 10 and $20 \mathrm{Gchips} / \mathrm{s}$ in presence of electronic dispersion compensation, and Fig. $2 \mathrm{~b}$ shows ONU launch power versus PON reach for different number of simultaneous users. In our simulation, QPSK is used as a modulation scheme at the transmitter side, all ONUs use the same laser at $1550 \mathrm{~nm}$ wavelength. We also consider a standard single mode fiber (SMF-28). All noise effects are considered at the receiver. Local oscillator power at the OLT is set to $0 \mathrm{dBm}$, iterative decoder iterations are set to 10 iterations, and electronic dispersion post-compensation is also considered at the receiver. For Fig. 2a we vary the length of the spreading codes from 16 to 128 chips, either to accommodate more users (long spreading code) or to achieve higher bit rate (short spreading code). PON reach is fixed at $100 \mathrm{~km}$. For Fig. 2b, the information block length is set to 256 bits, the maximum chip rate is $10 \mathrm{Gchips} / \mathrm{s}$ and the spreading code length is 64 chips. We consider forward-error correcting (FEC) codes with $10^{-3}$ BER threshold.

It is observed from Fig. 2a that there is a trade off between increasing number of ONUs and increasing user bit rate, as expected. For example, for $20 \mathrm{Gchips} / \mathrm{s}$ curve, to achieve $500 \mathrm{Mbps}$ bit rate, OIDMA can accommodate 150 simultaneous users for $100 \mathrm{~km}$ reach. However, when increasing the bit rate to $1.25 \mathrm{Gbps}$, the number of ONUs is limited to only 54 ONUs. Also, for $10 \mathrm{Gchip} / \mathrm{s}$ curve, we observe that OIDMA can either accommodate 225 ONUs at 156.25 Mbps, or achieve a high bit rate of $1.25 \mathrm{Gbps}$ but limited to only 27 ONUs. Inset figure shows the constellation diagram of the received electrical signal before OIDMA decoder for 100 users at $-5 \mathrm{dBm}$ launch power, that is messed up due to the presence of noise effects, multiple access interference, dispersion, and non-linear effects.

Fig. $2 b$ shows the launch power versus PON reach for different number of users in the system with and without dispersion compensation. As expected, at relatively low launch power, the PON reach increases linearly as the launch power is increased. In such a linear regime the power simply compensates the fiber losses to achieve the same BER performance. For example, for 100 ONUs curve without dispersion compensation, at PON reach of $100 \mathrm{~km}$, ONU launch power can be as low as $-8 \mathrm{dBm}$ to achieve the FEC threshold. Increasing the power further enhances the BER performance till reaching high power levels, where nonlinear effects are not anymore negligible. Also, in presence of dispersion compensation ONU launch power can be even lower to reach $-11 \mathrm{dBm}$ at $100 \mathrm{~km}$ reach. At high power levels, it is the mutual interaction between group velocity dispersion and self phase modulation that governs the performance, and adding dispersion compensation introduces more dispersion as we are working in anomalousdispersion regime [7]. 
In addition, from another perspective electronic dispersion compensation extends the PON reach. For example, 100 ONUs OIDMA without dispersion compensation is limited to $108 \mathrm{~km}$ due to dispersion in fiber, but with dispersion compensation PON reach can be extended to $165 \mathrm{~km}$. That is an increase of about 53\% in PON reach. Also, for 110 ONUs we observe an increase in PON reach due to electronic dispersion compensation of about $25 \mathrm{~km}$.

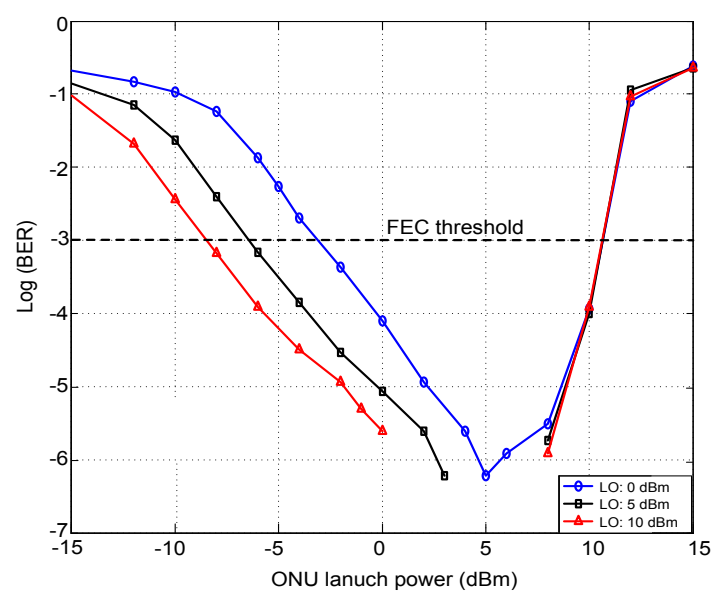

(a)

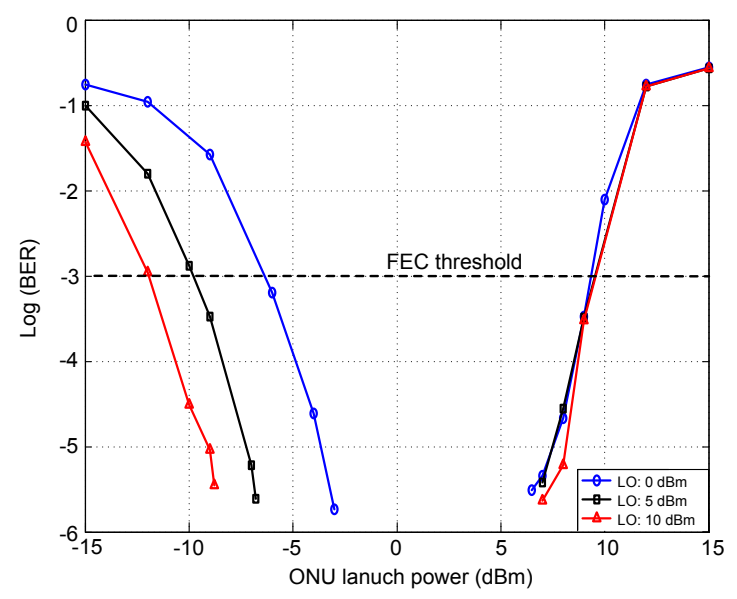

(b)

Fig. 3. BER 64 user OIDMA system for $150 \mathrm{~km}$ reach versus ONU launch power (a) without dispersion compensation and (b)with electronic dispersion compensation.

Fig .3a and Fig. 3b show the BER performance of 64 users OIDMA system for $150 \mathrm{~km}$ PON reach versus ONU lanuch power with and without electronic dispersion compensation respectively. It is observed from Fig. 3a that increasing local oscillator power at the OLT improves the BER performance considerably, and consequently, increases ONU launch power range and still achieving the FEC threshold. It is intuitive that adding electronic dispersion compensation improves the BER performance as shown in Fig. $3 b$.

\section{Conclusion}

A coherent optical interleave-division multiple-access (OIDMA) PON uplink scenario with electronic dispersion compensation has been proposed. Our results reveal that coherent OIDMA can be deployed in long reach PONs with large number of users at a reasonably low launch power. Adding electronic dispersion compensation increases PON reach and further enhances the performance. As a result, OIDMA is a rival to PON multiple access techniques in PON reach and ONU lanuch power perspectives.

\section{References}

1. L. G. Kazovsky, W.-T. Shaw, D. Gutierrez, N. Cheng, and S.-W. Wong, "Next-generation optical access networks," Journal of Lightwave Technology 25, no. 11 (2007): 3428-3442.

2. J. A. Salehi, "Code division multiple access techniques in optical fiber networks, Part I: Fundamental principles," IEEE Trans. Commun., vol.37, pp. 824833, Aug. 1989.

3. N. Cvijetic, " OFDM for Next-Generation Optical Access Networks," IEEE/OSA J. Lightwave Technol., vol. 30, issue 4, 2012, pp. 384-398.

4. E. A. El-Fiky, A. M. Morsy, Ziad A. El-Sahn, and Hossam M. H. Shalaby, "Coherent OIDMA technique for next generation long reach PONs," inPROC. CLEO 2013, San Jose, CA, June 9-14, 2013.

5. A. M. Morsy, E. A. El-Fiky,and Hossam M. H. Shalaby, "Performance Analysis and Comparison of Optical IDMA and Optical CDMA Techniques Using Unipolar Transmission Scheme," accepted at NOC 2013, GRAZ, Austria, July 10-12, 2013.

6. Li Ping, L. Liu, K. Y. Wu, and W. K. Leung, "Interleave-division multiple-access (IDMA) communications,"in Proc. 3rd International Symposium on Turbo Codes and Related Topics, 2003, pp. 173180.

7. G.Agrawal, "Nonlinear Fiber Optics," third edition, Academic Press, 2001. 